\title{
LAVORI PUBBLICATI
}

\author{
DAL PROFESSORE ANGELO BATTELLI \\ Elenco compilato dal Prof. A. Naccari.
}

1. «Sui sistemi catottrici centrati ». Atti Torino XIX, 297, 1884. - Repert. d. Phys. 1885.

2. « Sulle proprietà termoelettriche delle leghe ». Parte T. Mem. Torino XXXVI, 487, 1884.

3. «Sulla propagazione della luce in un sistema catadiottrico ». Atti Ist. Ven. (6) II, 1081, 1885.

4. "Sull'aberrazione di sfericità nei telescopi di Grégori e Cassegrain 》. Atti Torino XX, 670, 1885.

5. Con S. Pagliani. «Sull'attrito interno dei liquidi ». Atti Torino XX, 449 e 653, 1885.

6. "Conseguenze d' una nuova ipotesi di Kohlrausch sui fenomeni termoelettrici ». Rend. Line. (6) I, 117, 1885. N. C. XVIII, 219, 1885.

7. Con A. Naccari. «Sul fenomeno Peltier nei liquidi ». Nota I e II. Atti Torino XX, 825 e 964, 1885.

8 . « Sui fenomeni termici che accompagnano la formazione dei miscugli di sostanze non metalliche ». Rend. Linc. (4) I, 646, 1885.

9. «Influenza della pressione sulla temperatura di fusione di alcune sostanze ». Atti Ist. Ven. (6) III, 1781, 1886. N. C. XIX, 232, 1886. 
10. Con L. Palazzo. « Sulle variazioni di volume di alcuni corpi per effetto della fusione ». Mem. Linc. (4) I, 283, 1885 .

11. Con M. Martinettit. "Sui calori specifici e di fusione di sostanze non metalliche ». Rend. Linc. (4) I, 621, 1885.

12. Con L. Palazzo. "Intorno alla fusione dei miscugli di alcune sostanze non metalliche ». Parte I. Atti Torino XIX, 514, 1884.

13. Idem. "Intorno alla fusione dei miscugli binari di sostanze non metalliche ». Atti Torino XX, 1058, 1885.

14. Con M. Martinetri. "Sulla fusione di miscugli binari di sostanze non metalliche ». Parte II. Atti Torino, $\mathrm{XX}, 844,1885$.

15. Idem. «Sulla variazione di volume che si avvera nell'atto della mescolanza di sostanze organiche ». Rend. Linc. (4) II, $2 .^{\circ} 247,1886$.

16. Con A. Naccari. « Sul fenomeno Peltier nei liquidi ». Nota III. Atti Torino XXI, 581, 1886.

17. Idem. Sullo stesso argomento. N. C. XX, 201, 1886.

18. «Intorno all' influenza della magnetizzazione sopra la conducibilità termica del ferro ». Atti Torino XXI, 559, 1886.

19. Con M. Martinetti. «Un regolatore per la pressione dei gas ». L' Ingegneria civile e le arti industriali XII, settembre 1886.

20. «Sul fenomeno Thomson ». Studio sperimentale. Nota 1. Atti Torino XXII, 48, 1886. - N. C. 21, 228, 1887.

21. "Sul fenomeno Thomson ». Nota II. Atti Torino XXII, $369,1887$. - N. C. 22,157 e $221,1887$.

22. «Sul fenomeno Thomson nel piombo». Rend. Linc. (4) III, $1.0^{\circ} 212,1887$. - N. C. XXI, 250, 1887.

23. «Sul fenomeno Thomson nel nichel». Rend. Linc. (4) IIT, 2. $105,1887$.

24. «Sulle proprietà termoelettriche delle leghe ». Parte II. Atti Ist. Ven. (6) V, 1237, 1887.

25. « Sulla termoelettricità del mercurio ». Rend. Linc. (4) III, 6, 1887.

26. «Sulla termoelettricità delle amalgame ». Rend. Linc. (4) III, $2 .^{\circ} 37,1887$. 
27. «Sull' annullarsi del fenomeno Peltier al punto neutrale di alcune leghe ». Rend. Linc. (4) III, 1. ${ }^{\circ} 404,1887$. N. C. XXIII, 64, 1888.

28. «Sulla resistenza elettrica delle amalgame». Mem. Linc. (4) IV, 206, 1887.

29. «Sulle variazioni della resistenza elettrica e del potere termoelettrico del nichel al variare della temperatura ». Atti Torino XXIII, 169, 1888. - N. C. XXXIV, 125, 1893.

30. «Sulle correnti telluriche ». Nota preliminare. Rend. Linc. (4) IV, 2. 25,1888 . - N. C. XXIV, 45, 1888.

31. «Sul fenomeno Peltier a diverse temperature e sulle sue relazioni col fenomeno Thomson ». Mem. Linc. (4) V, 632, 1888. - N. C. XXVII, 111, 1890.

32. «Misure assolute dell'inclinazione magnetica nella Svizzera ». Rend. Linc. (4) V, 1.0 771, 1889.

33. «Sulle proprietà termiche dei vapori ». Parte I. Mem. Torino XL, 21, 1889. - N. C. XXX, 235, 1891.

34. « Misure assolute degli elementi del magnetismo terrestre eseguite nella Svizzera nel 1889 ». Rend. Iinc. (4) VI, $10^{\circ} 513,1890$.

35. « Sulle correnti telluriche ». Annali Uff. Centr. Met. IX, 1, 1887. - N. C. XXVII, 233, 1890; XXVIII, 97, 1890.

36. « Sull' evaporazione dell'acqua e del terreno umido ». Annali Uff. Centr. Met. 9, 99, 1887. - N. C. XXVIII, 247, 1890.

37. «Sull'influenza della forza elettromotrice degli elettrodi nello studio delle correnti telluriche ». Rend. Linc. (4) VII, 1. $403,1891$.

38. "Misure assolute degli elementi del magnetismo terrestre nella Svizzera eseguite nel 1888 e 1889 ». Annali Uff. Centr. Met. XI, 29, 1889.

39. « Sul crepuscolo» N. C. XXIX, 97, 1891.

40. «Sulle proprieta termiche dei vapori ». Parte II. Mem. Torino XLI, 25, 1890. - N. C. XXXI, 156, 1892; XXXII, 38, 1892.

41. «Sulle proprietà termiche dei vapori 》 Parte III. Mem. Torino XLII, 119, 1892. - N. O. XXXIV, 97, 1893. 
42. « Misure per la costruzione della carta magnetica della Svizzera ». Parte I, II, III. Atti Ist. Ven. (7) III, 455, 973, 1479, 1892. - N. C. XXXII, 250, 1892.

43. "Sullo stato della materia nel punto critico ». Parte I. Atti Ist. Ven. (7) III, 1615, 1893. - N. C. XXXIII, 22 e $57,1893$.

44. « Sulle proprietà termiche dei vapori 》. Parte IV. Mem. Torino XLIII, 99, 1893. - N. C. XXXIV, 186, 1893.

45. « Carta magnetica della Svizzera ». Annali Uff. Centr. Met. XIV, 83, 1892.

46. «Sulle isobare dei vapori ». Rend. Linc. (5) II, $1 .^{\circ}$ 171, 1893. - N. C. XXXIV, 5, 1893.

47. «Sullo stato della materia nel punto critico ». Parte II. Atti Ist. Ven. (7) IV, 685, 1893.

48. «Influenza del magnetismo e delle azioni meccaniche sul fenomeno Thomson ». Atti Ist. Ven. (7) IV, 1452, 1893. N. C. XXXV, 55, 1894.

49. «Influenza del magnetismo e delle azioni meccaniche sul fenomeno Peltier ». Atti Ist. Ven. IV, 1581 e 1637, 1893.

50. « Influenza del magnetismo e delle azioni meccaniche sulle correnti termoelettriche ». Atti Ist. Ven. IV, $1676 \mathrm{e}$ $1745,1893$.

51. « Sul comportamento termoelettrico dei metalli magnetizzati ». Rend. Linc. (5) II, 162, 1893.

52. «Sur les variations seculaires des elements du magnetisme terrestre en Suisse ». Arch. 'des Sc. Phys. et Nat. XXVIII, 202, 1892.

.53. « Influenza del magnetismo e delle azioni meccaniche sui fenomeni termoelettrici ». N. C. XXXV, 55, 1894.

54. " On the thermal behaviour of liquids ». Phil. Mag. XXXVIII, 245, 1894.

55. « Sulle proprietà termiche dei vapori ». Parte V. Mem. Torino XLIV, 57, 1894. - N. C. I, 230, 1895.

56. « Sulle proprietà termiche dei vapori 》. Parte VI. Mem. Torino, 45, 235, 1896. - N. C. II, 97, 1895.

57. « Sul luogo di emanazione dei raggi di Röntgen nei tubi a vuoto ». N. O. III, $129,1896$. 
58. « Ricerche sulle azioni fotografiche nell'interno dei tubi di scarica ». N. C. III, 193, 1896.

59. Con A. Garbasso «Sopra i raggi del Röntgen ». N. C. III, 40, 1896.

60. Idem «Sopra un modo per ridurre il tempo di posa delle fotografie eseguite coi raggi Röntgen ». N. C. III, $167,1896$.

61. Idem "Raggi catodici e raggi $X$ ». N. C. III, 289, 1896.

62. Idem « Sulla dispersione delle cariche elettrostatiche prodotta dai raggi ultravioletti ». N. C. III, 321, 1896.

63. Idem « Azione dei raggi catodici sopra i conduttori isolati ». N. C. IV, 129, 1896.

64. Idem «Sur quelques faits se rapportants aux rayons de Röntgen ». C. R. CXXII, 603, 1896.

65. "Rapporti fra le azioni fotografiche all'interno e all'esterno dei tubi a vuoto ». N. C. V, 169, 1897.

66. « Rapporti fra i raggi catodici e i raggi del Röntgen ». N. C. V, 386, 1897. - Phil. Mag. XLV, 163, 1898.

67. « Metodi e conquiste della fisica ». Discorso letto per l'inaugurazione dell'anno accademico. Annuario della R. Università di Pisa 1897-98.

68. Con A. Garbasso « Azione dei raggi catodici sopra i conduttori isolati ». N. C. VI, 5, 1897.

69. «Effluvi elettrici unipolari nei gas rarefatti ». N. C. VII, $81,1898$.

70. Con F. BatTelli « Trattato pratico per le ricerche di elettricità in medicina ». Roma, Soc. Ed. Dante Alighieri, 1898.

71. Con A. Stefanini « Ricerche crioscopiche ed ebullioscopiche ». N. C. IX, 5, 1899.

72. Idem «Sulla velocità dei raggi catodici e sulla conduttività elettrolitica dei gas ». N. C. X, 324, 1899. Phys. Zeits. IV, 51, 1899.

73. Idem « Esposizione critica della teoria della dissociazione elettrolitica ». Lucea, Baroni, 1899.

74. Con M. Pandolfi «Sull' illuminazione dei liquidi ». N. C. IX, 321, 1899. 
75. Con L. Magrr «Sui raggi anodici e sui raggi catodici ». N. C. X, 264, 1899. - Phys. Zeits. II, 18, 1899.

76. «La chaleur spécifique des gaz ». Rapport presenté au Congrès international de Physique à Paris en 1900. N. C. XII, 300, 1900.

77. «Ricerche sulla legge di Boyle a pressioni molto basse 》. Parte I. N. C. I, 5, 1901. - Phys. Zeits. II, 409, 1901. Ann. de Ohim. et Phys. XXV, 38, 1912.

78. «Sulla legge di Boyle a pressioni molto basse ». Parte II. N. C. I, 81, 1901 - Phys. Zeits. III, 17, 1901. - Ann. de Chim. et Phys. XXV, 38, 1902.

79. « Die spezifische Wärme der Gase ». Phys. Zeits. II, $376,1901$.

80. « Riccardo Felici ». Commemorazione letta nella VI riunione della Società italiana di fisica. - N. C. 4, 233, 1902.

81. Con L. Magri «Sulle scariche oscillatorie ». Mem. Torino XLI, 335, 1902.

82. Idem « Sulle scariche oscillatorie». Parte I. N. C. III, 177, 1902. - Phys. Zeits. III, 539, 1902.

83. Idem. Sullo stesso argomento. Parte II. N. C. III, 257, 1902. - Phys. Zeits. VI, 181, 1902.

84. Con P. Cardani « Trattato di fisica sperimentale ad uso delle università ». Vol. I: Meccanica - Metrologia - Proprietà dei solidi, dei liquidi, dei gas. Milano, Vallardi, 1902.

85. Con L. Magri « Les decharges oscillatoires 》. Arch. des Sc. Phys. et Nat. VII, 5, 1903; VIII, 139, 1903.

86. Con F. MaCCarrone « Se le emanazioni radioattive sieno elettrizzate ». Rend. Linc. XIII, 539, 1904. - N. C. VII, 259, 1904.

87. Con A. Stefanini « Sulla natura della pressione osmotica ». Rend. Linc. XIV, 1." 669, 1905. - N. C. X, 137, 1905. - Phys. Zeits. VII, 190, 1906. - Journ. de Phys. VI, 407, 1907.

88. «Resistenza elettrica dei solenoidi per correnti di alta frequenza ». Rend. Linc. XV, 148, 1906. - Phys. Zeits. VIII, 296, 1907. - Journ. de Phys. VI, 559, 1907. 
89. Sullo stesso argomento. Nota II. Rend. Linc. XV, 471, 1906. - Phys. Zeits. VIII, 530, 1907. - Journ. de Phys. VI, 701, 1907.

90. Sullo stesso argomento. Nota III. Rend. Linc. XV, 529, 1906. - Phys. Zeits. VIII, 533, 1907. - Journ. de Phys. VII, 62, 1908.

91. «Ricerche sperimentali sulla resistenza dei solenoidi alle correnti di alta frequenza ». Nota IV. Rend. Linc. XV, 255, 2.0 1916. - Phys. Zeits. VIII, 809, 1907.

92. « Ricerche teoriche e sperimentali sulla resistenza dei solenoidi per correnti di alta frequenza ». N. C. XI, 285, 1906. - Phys. Zeits. IX, 154, 1908.

93. «Con L. Magri « La scarica oscillatoria neì fili di ferro ». Rend. Linc. XV, 63, 2.0 1906. - Phys. Zeits. VIII, 298, 1907.

94. Idem « La scarica oscillatoria nei solenoidi con anima di ferro ». Rend. Linc. XV, 397, 2.० 1906.

95. Idem « $L$ 'isteresi magnetica del ferro per correnti di alta frequenza ». Rend. Linc. XV, 2. 1906.

96. Idem « Sulle scariche oscillatorie ». Parte IIT. N. C. XII, $193,1906$.

97. Con A. Occhialior e S. Chella « Studi di radioatti vità 》. Rend. Linc. XV, 262, 2.0 1906. - N. C. XII, 281, 1906. - Phys. Zeits. VIII, 65, 1907. - Journ. de Phys. VI, 899, 1907.

98. « Le energie radianti ». Nel volume « Il pensiero mo derno ». Milano, Treves, 1907.

99. « Calori specifici dei liquidi che solidificano a temperatura bassa 》. Rend. Linc. XVI, $243,1 .^{\circ} 1907$ - N. C. XIIT, 418, 1907. - Journ. de Phys. VII, 881, 1907. Phys. Zeits. IX, 671, 1908.

100. Con A. Stefanini \& Relazione fra la pressione osmotica e la tensione superficiale ». Rend. Linc. XVI, $111^{\circ}$ 1907. - N. C. XIII, 15, 1907. - Journ. de Phys. VIII, 949, 1908.

101. Idem « Sulla relazione fra la tensione superficiale e la pressione osmotica ». Rend. Line. XVI, 663, 2. 1907. 
102. Con I. Magri « La scintilla elettrica nel campo magnetico ». Rend. Linc. XVI, 155, 1. 1907. - N. C. XIII, $263,1907$.

103. Idem « Comportamento dei vapori metallici nella scintilla elettriea 》 Rend. Linc. XVI, 12, 2. ${ }^{\circ}$ 1907. - N. C. $\mathrm{XV}, 188,1908$.

104. «Sulla resistenza elettrica dei solenoidi per correnti ad alta frequenza ». Rend. Linc. XVII, 61, 1. 1908.

105. « L' opera scientifica di Evangelista 'Torricelli ». Faenza, 1908.

106. Con L. Magri « Sullo spettro della scintilla elettrica ». Rend. Linc. XVII, 391, 1. 1908.

107. Con A. Occhialini e $\mathbf{S}$. Chella. « La radioattività 》.

Bari, Laterza, 1909. - Leipzig, J. A. Barth, 1910. Paris, Gauthier-Villars, 1910.

108. « La navigazione aerea ». Rivista nautica, N. 1, 1910.

109. « Il problema della dirigibilita, ». Ivi, N. 3 .

110. «I dirigibili ». Ivi, N. 5 .

111. « Il problema dell' aviazione ». Ivi, N. 6.

112. «Gli aeroplani ». Ivi, N. 7.

113. « La resistenza dell' aria ». Ivi, N. 10.

114. « Breve esame critico dei vari sistemi di navigazione aerea ». Ivi, N. 12.

115. «Carlo Matteucci ». Discorso commemorativo tenuto a Forlì nel $10^{\circ}$ centenario della nascita. Forlì, Coop. Tip. Forlivese, 1911.

116. «Corso di Fisica per i licei ». 2 vol. Milano, Pallestrini, 1911.

117. «Corso di Chimica per i licei ». 1 vol. Milano, Pallestrini, 1911.

118. "Corso di Fisica per gli istituti tecnici ». 3 vol. Bologna, Zanichelli, 1911.

119. "L' opera di Alessandro Volta ». Rivista delle comunicazioni, fase. $3 .^{\circ}$, Marzo 1912.

120. Con A. Razzauti « Corso di Scienze Fisiche e Naturali per le scuole normali ». 3 vol. Roma, Boscaini, 1913. 
121. Con P. Cardani. «Trattato di Fisica sperimentale ad uso delle universitì ». Vol. II: Acustica e Ottica. Milano, Vallardi, 1913.

12\%. «Corso di Fisica e Chimica per i licei moderni 》. 3 vol. Bologna, Zanichelli, 1914.

12:3. Con A. Razzauti « Corso di Scienze Fisiche e Naturali per le scuole complementari ». 3 vol. Roma, Boscaini, 1914.

1\%4. Idem «Corso di Scienze Fisiche e Naturali per le seuole tecniche ». 2 vol. Roma, Boseaini, 1915.

125. Con P. Cardani. "Trattato di Fisica sperimentale ad uso delle università ». Yol. III : Calore. Milano, Vallardi, 1916. 